\title{
Spinal perimedullary arteriovenous fistula supplied by the artery of Desproges-Gotteron: A case report with literature review
}

\author{
YIHENG WANG and JINLU YU \\ Department of Neurosurgery, The First Hospital of Jilin University, Changchun, Jilin 130021, P.R. China
}

Received November 17, 2021; Accepted December 23, 2021

DOI: $10.3892 / \mathrm{mi} .2021 .26$

\begin{abstract}
Spinal perimedullary arteriovenous fistula (PAVF) of the conus medullaris and cauda equina supplied by the artery of Desproges-Gotteron (ADG) is rare. The present study reports such a rare case and also presents a brief literature review. The patient described herein was a 31-year-old female. She suffered sudden weakness in the lower limbs, a condition which was then gradually aggravated. A physical examination revealed paraplegia, and urination and defecation difficulties. Spinal magnetic resonance imaging (MRI) and computed tomography angiography (CTA) revealed extensive perimedullary vein dilation with the involvement of the ADG as the main feeding artery. Digital subtraction angiography (DSA) confirmed a high-flow PAVF supplied by the ADG and artery of Adamkiewicz (AKA). Microsurgical treatment under intraoperative DSA assistance was performed. The PAVF was resected, and immediately following DSA confirmed the complete removal of the PAVF. Following discharge, the patient was prescribed rehabilitation exercises and gradually recovered. A follow-up CTA and MRI confirmed that the PAVF was cured, and her modified Rankin scale score was 2. Therefore, as demonstrated herein, microsurgery with intraoperative DSA assistance may be considered an effective treatment option for PAVFs with ADG.
\end{abstract}

\section{Introduction}

Spinal perimedullary arteriovenous fistula (PAVF) is an uncommon arteriovenous shunt of the conus medullaris

Correspondence to: Dr Jinlu Yu, Department of Neurosurgery, The First Hospital of Jilin University, 1 Xinmin Avenue, Changchun, Jilin 130021, P.R. China

E-mail: jlyu@jlu.edu.cn

Abbreviations: ADG, artery of Desproges-Gotteron; AKA, artery of Adamkiewicz; CTA, computed tomography angiography; DSA, digital subtraction angiography; MRI, magnetic resonance imaging; PAVF, perimedullary arteriovenous fistula

Key words: perimedullary arteriovenous fistula, artery of Desproges-Gotteron, treatment and cauda equina (1). PAVFs at this location are often only supplied by the thoracolumbar radiculomedullary artery (2). The artery of Desproges-Gotteron (ADG) may arise from the internal iliac artery or the iliolumbar artery up to the conus medullaris (3). In rare cases, the ADG can be involved as the feeding artery of the PAVF (4).

The present study reports the case of a female patient with PAVF supplied by the ADG. As a PAVF feeding artery, the ADG can be missed upon angiography. The spinal cord has a complex origin of the feeding artery (Fig. 1). Therefore, the understanding of spinal cord vascular disease is often difficult; thus, reports of such cases are of utmost importance. In addition, the present study also performed a brief literature review of this condition in order to shed further light into this rare occurrence.

\section{Case report}

A 30-year-old female was admitted to the First Hospital of Jilin University (Changchun, China) on September 23, 2019. She was in a good health prior to her admission. At 3 days prior to her admission, she suffered a sudden weakness of the lower left limb, and the symptoms gradually became aggravated. At 2 days following symptom onset, she suffered paraplegia, as well as difficulties in urination and defecation. A physical examination revealed that she had a muscle strength of grade 1 in the right lower limb and grade 2 in the left lower limb (5). Superficial and deep sensations were reduced in the bilateral lower limbs. The sensory level was at the T12 and L1 level (6). The Babinski sign was negative in the left lower limb and positive in the right lower limb.

Spinal magnetic resonance imaging (MRI) and computed tomography angiography (CTA) revealed extensive intradural extramedullary flow void signs in the spinal canal, the dilation of the spinal vein extending upward to a high cervical level, and the involvement of the ADG and artery of Adamkiewicz (AKA) as the main feeding arteries. The location of the vascular lesion was at the conus medullaris and cauda equina and was filled with the spinal canal. The length of the whole spinal cord was measured using a GE Workstation (version 4.7; GE Healthcare; Cytiva) and was $39 \mathrm{~cm}$. The pre-operative data are presented in Fig. 2. Microsurgical treatment with intraoperative digital subtraction angiography (DSA) assistance was planned. The intraoperative DSA revealed a PAVF at the L1 level supplied by the right AKA and the left ADG (Fig. 3). 


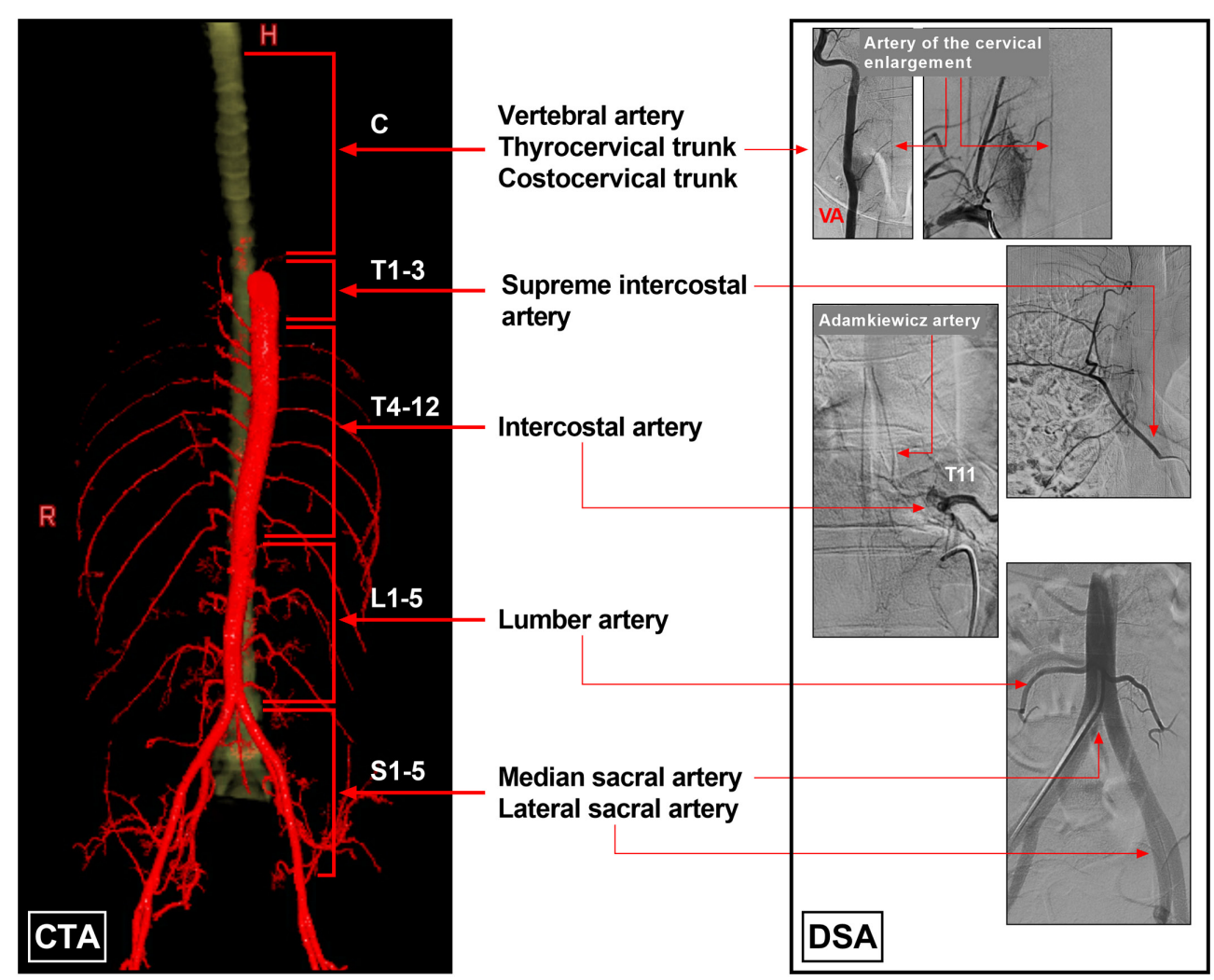

Figure 1. Blood supply system of the spinal cord. The left section of the image was created using CTA imaging, and the blood supply of the spinal cord was divided into five parts as follows: Cervical arteries (vertebral artery, thyrocervical trunk and costocervical trunk), supreme intercostal artery, intercostal artery, lumbar artery, and median and lateral sacral artery. The right section of the image was established using DSA imaging and illustrates spinal arteries and their origins. C cervical vertebra; CTA, computed tomography angiography; DSA, digital subtraction angiography; H, head; L, lumbar vertebra; R, right; S, sacral vertebra; $\mathrm{T}$, thoracic vertebra.
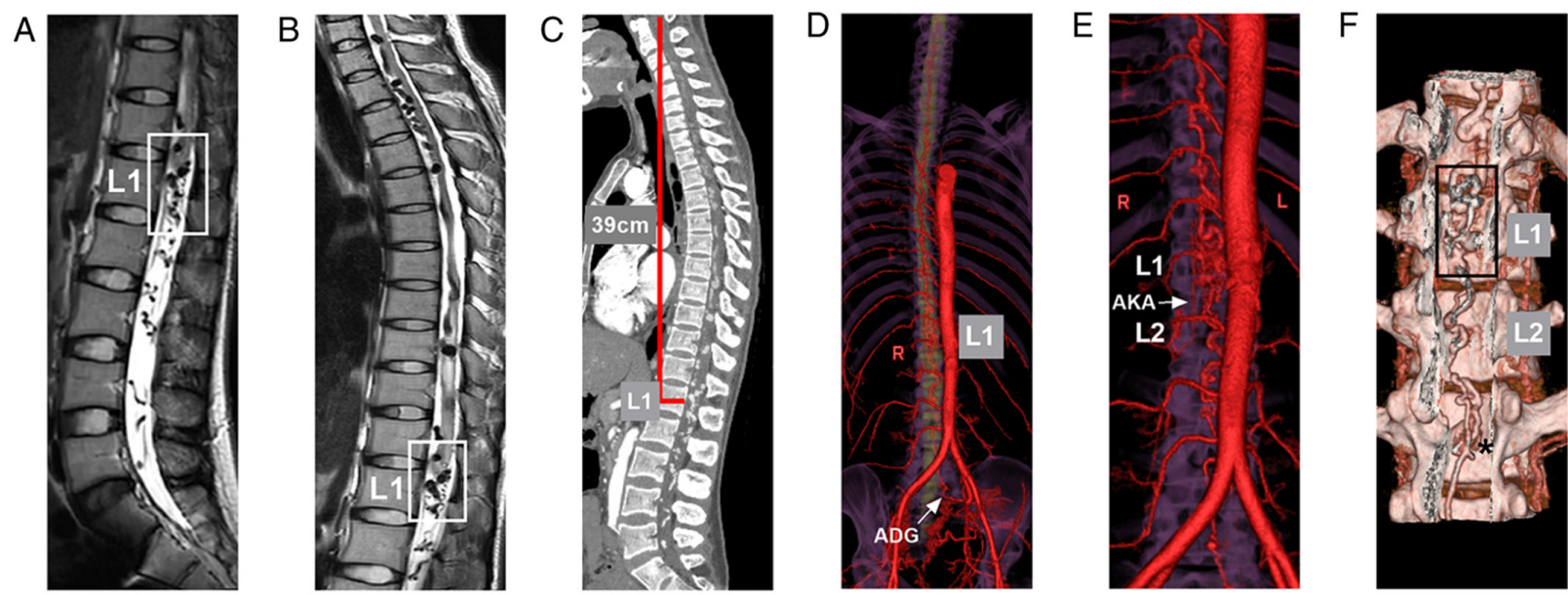

Figure 2. Pre-operative MRI and CTA. (A Lumber and (B) thoracolumbar T2-weighted MRI illustrating extensive intradural extramedullary flow void signs. The frames indicate the vascular lesion located at the conus medullaris and cauda equina nerves (L1 level). (C) CTA of maximum intensity projection revealed an extensive contrasted lesion in the whole spinal canal. In the GE Workstation (version 4.7), the cured three-dimensional length of the spinal canal from the atlas to the L1 level (the same as the length of the spinal cord) was $39 \mathrm{~cm}$, which was measured using the two-click AVA tool. (D) Reconstructive CTA of the spinal cord arteries illustrating a vascular lesion at the L1 level, with the dilation of the whole spinal vein extending upward to a high cervical level from the L1 level, and the involvement of the ADG from the left internal iliac artery as the main feeding artery (arrow). (E) Reconstructive CTA of the spinal cord arteries illustrating the right AKA (arrow) from the lumbar artery at the L2 level also supplied the vascular lesion. (F) Reconstructive CTA with the bone and vessel illustrating the vascular lesion located at the L1 level (frame), and the ADG divided into two branches (asterisk) into the lesion. ADG, artery of Desproges-Gotteron; AKA, artery of Adamkiewicz; CTA, computed tomography angiography; L, left; L1, first lumbar vertebra; L2, second lumbar vertebra; MRI, magnetic resonance imaging; $R$, right.

The surgery was centered on the level of the L1 vertebral body, and the spinous processes and lamina of the T12 and
L1-3 segments were removed. After opening the spinal dura, the PAVF was found, presenting with dilated and tortuous 

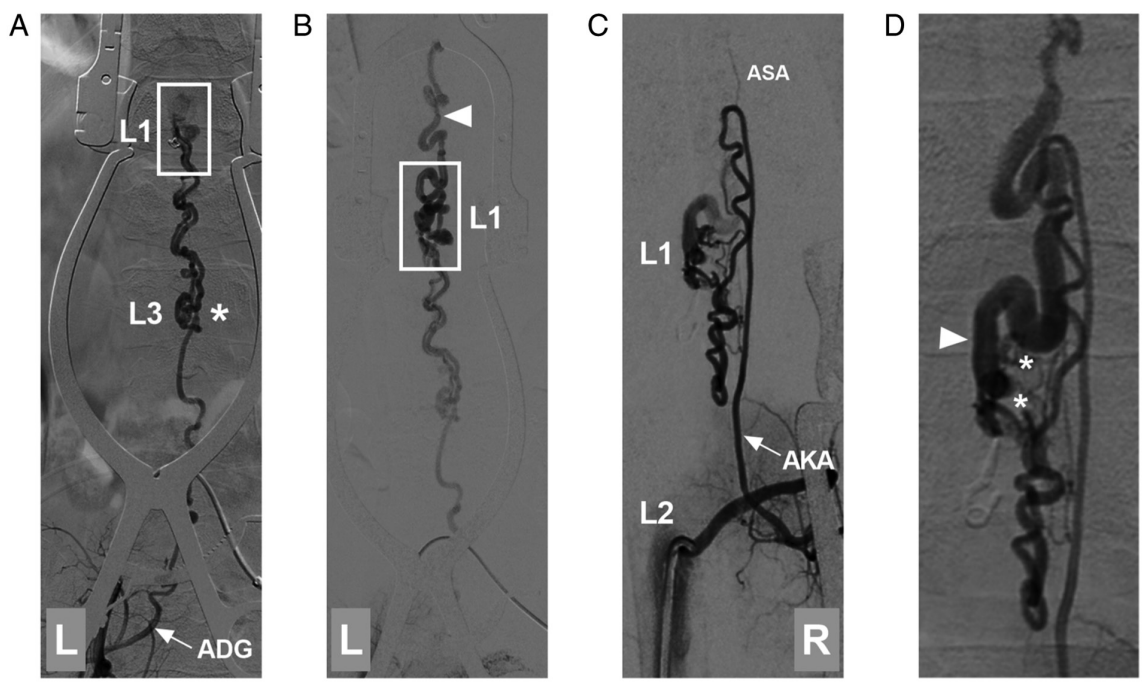

Figure 3. DSA prior to PAVF removal. (A) Arterial-phase DSA of the left iliac artery illustrating that the ADG was from the internal iliac artery as the feeding artery to the PAVF (frame at L1). At the L3 level, the ADG was divided into two branches (asterisk). (B) Late arterial-phase DSA illustrating that the PAVF at the L1 level (frame) transferred into the draining vein (arrowhead). (C) Arterial-phase DSA of the lumbar artery (L2 level) showing that the PAVF was supplied by the right AKA (arrow); a hypoplastic ASA was found. (D) Late arterial-phase DSA illustrating the PAVF architecture, in which the asterisks indicate multiple slims feeding arteries directly into a large draining vein (arrowhead). AKA, artery of Adamkiewicz; ADG, artery of Desproges-Gotteron; ASA, anterior spinal artery; DSA, digital subtraction angiography; L, left; L1, first lumbar vertebra; L2, second lumbar vertebra; L3, third lumbar vertebra; PAVF, perimedullary arteriovenous fistula; $R$, right.
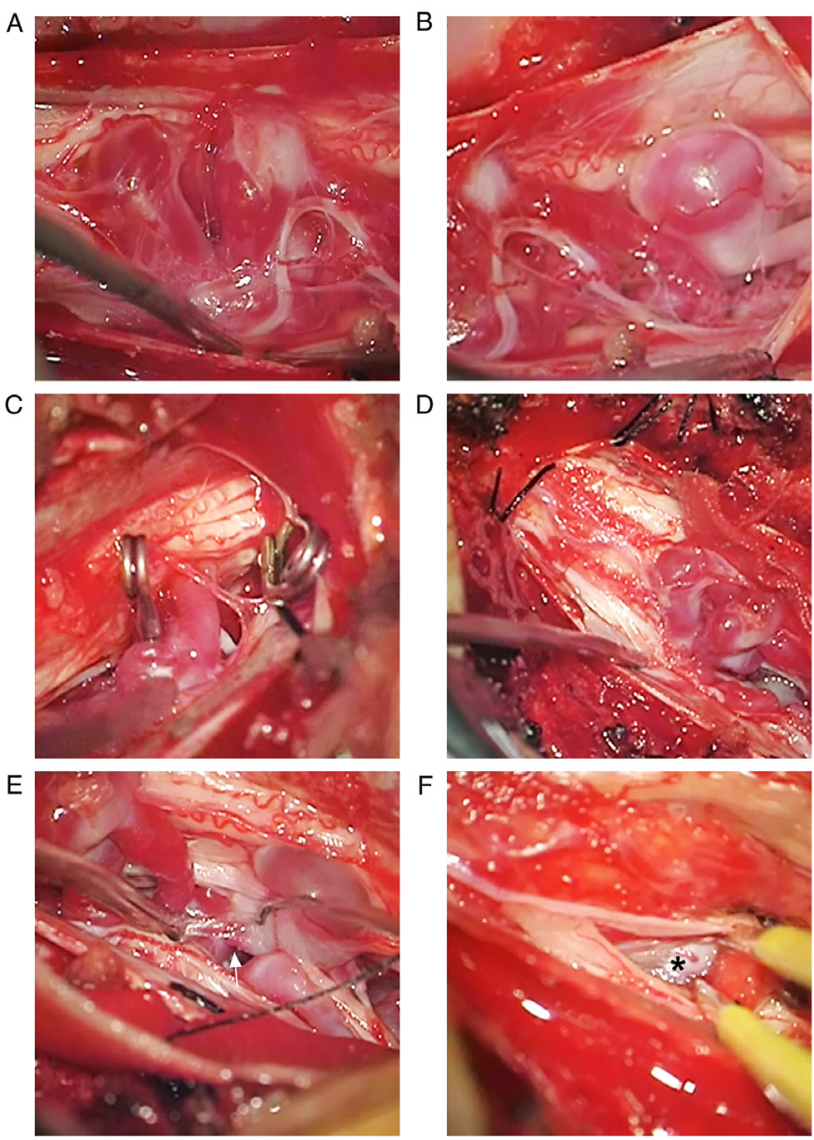

Figure 4. Intraoperative imaging of PAVF removal. (A and B) Intraoperative imaging prior to PAVF removal revealed tortuous vessels around the conus medullaris and cauda equina nerves. (C) Intraoperative imaging illustrating two deep feeding arteries of the PAVF from the artery of Desproges-Gotteron that were clipped. (D) Intraoperative imaging illustrating that the PAVF shrank after the two feeding arteries were cut. (E) Intraoperative imaging illustrating the feeding artery of the PAVF from the artery of Adamkiewicz (arrow); it was then cut. (F) Intraoperative imaging illustrating the large deep draining vein (asterisk). PAVF, perimedullary arteriovenous fistula. vessels on the surface of the conus medullaris and cauda equina nerves (Fig. 4A and B). An intraoperative angiography of the left iliac artery confirmed that two large feeding arteries from the ADG supplied the PAVF, and they were coagulated and cut (Fig. 4C and D). Subsequently, the angiography of the right lumbar artery revealed that the feeding artery from the AKA supplied the PAVF, and it was coagulated and cut (Fig. 4E). The PAVF was then removed; the draining vein is illustrated in Fig. 4F. The complete removal of the PAVF was confirmed upon an intraoperative DSA (Fig. 5A and B).

After the surgery, the patient's symptoms did not become aggravated, and anticoagulation therapy was administered to avoid thrombosis of the draining vein. A pathological examination revealed PAVF changes (Fig. 5C). Following discharge, she was prescribed rehabilitation exercises, including functional electrical stimulation and exercise therapy on motor control and functional ability of the lower extremity, and she gradually recovered. The 3-month follow-up CTA did not reveal a PAVF (Fig. 5D and E). At 2-years post-surgery, an MRI did not reveal a PAVF (Fig. 5F). Her modified Rankin scale score was 2 (7).

\section{Discussion}

The ADG is the spinal posterior radiculomedullary or radiculopial artery, also known as the 'cone artery'; this inconstant artery was first described by Desproges-Gotteron in $1955(4,8)$. The ADG may arise from the internal iliac artery or the iliolumbar artery and courses alongside the L5 or S1 nerve roots up to the conus medullaris and anastomoses with the perimedullary network (conal basket) (1).

The ADG is a rare arterial variation that functions as a feeder of spinal vascular malformations (1). Therefore, this artery is often missed, particularly when it is located near the contralateral iliac artery, and spinal angiography is not 


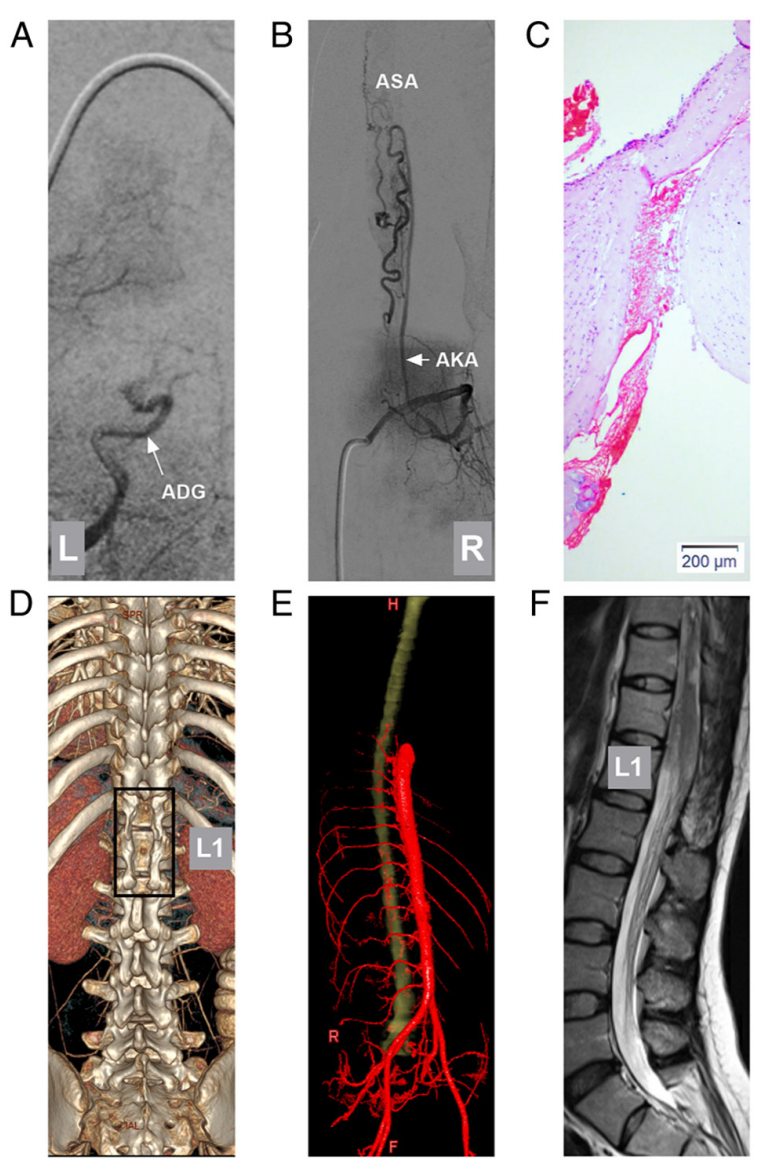

Figure 5. Post-operative DSA, pathology, follow-up CTA and MRI. (A) Post-operative DSA of the left iliac artery illustrating that the ADG (arrow) was occluded. (B) Post-operative DSA of the lumbar artery (L2 level) illustrating that the AKA (arrow) did not supply the PAVF, and a hypoplastic ASA was found. (C) Pathological evaluation illustrating that the PAVF was an irregular vessel with a dilated lumen and thickened wall. (D) The 3-month follow-up CTA illustrating the post-operative bone window (frame) centered on the L1 level. (E) The 3-month follow-up CTA illustrating no dilated draining vein. (F) The 2-year follow-up T2-weighted MRI illustrating the regression of the PAVF; dilated vessels were not found. AKA, artery of Adamkiewicz; ADG, artery of Desproges-Gotteron; CTA, computed tomography angiography; DSA, digital subtraction angiography; L1, first lumbar vertebra; MRI, magnetic resonance imaging; PAVF, perimedullary arteriovenous fistula.

performed. If the ADG is missed, treatment may be incomplete. Due to its rarity, the present study reports a case of a PAVF supplied by the ADG in an aim to share the experience with its treatment. At the same time, a search of the literature was performed on PubMed, which yielded only three cases (Table I).

Among these three cases, one was an arteriovenous malformation (4), one was a dural AVF (9) and one was a PAVF (1). In the case described herein, the patient had a PAVF. As shown in Table I, all patients, including the patient in the present study, were female, ranging in age from 8-65 years. PAVFs are considered type IV spinal vascular malformations and are located on the surface of the spinal cord without an intervening nidus of abnormal vessels (10). When PAVFs are ventral and directly fed by anterior spinal arteries, they are subpial in location, whereas those fed by posterior spinal arteries are subarachnoid.

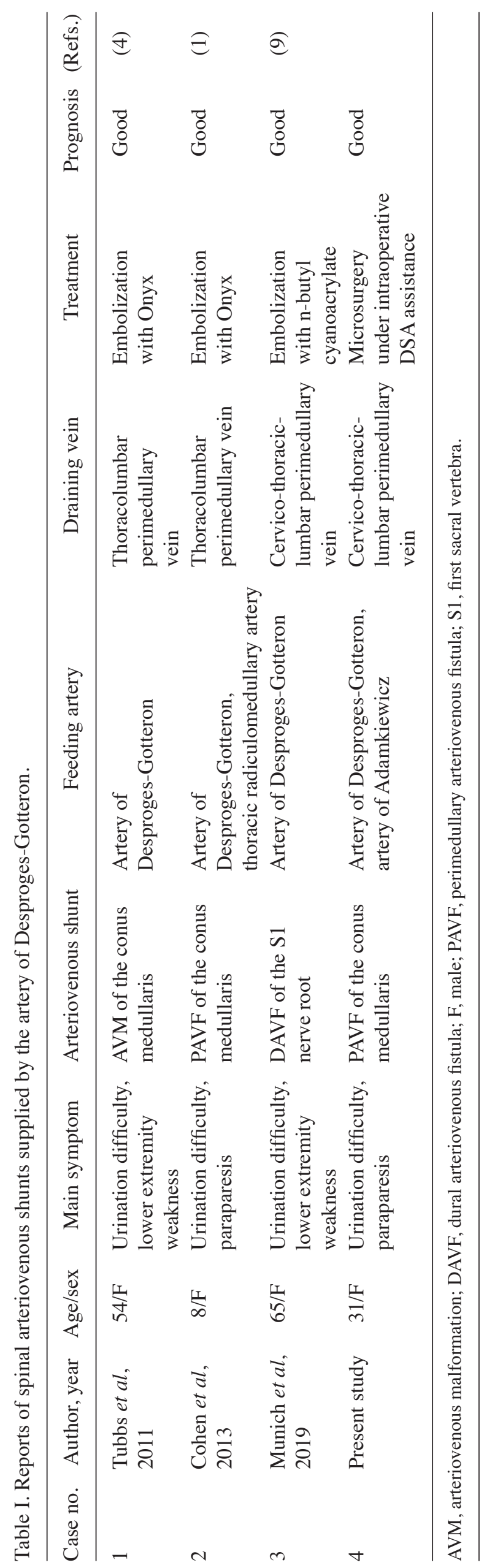


When PAVFs occur, the fistula shunts high-pressure arterial blood into the spinal cord vein system, which leads to pial venous reflux and congestion, causing myelopathy (11). PAVFs typically cause clinical symptoms, including a weakness of the lower extremities, urination difficulties and intradural subarachnoid hemorrhage. Of these symptoms, hemorrhage occurs in $36 \%$ of PAVFs, primarily high-flow PAVFs (10). The symptoms of PAVFs vary; in the present case, the high-flow PAVF did not result in bleeding, although it did result in neurological deficits, and the symptoms progressed very rapidly. Within 3 days, lower limb paralysis occurred due to the high-flow shunt, which tends to have a malignant natural history.

Currently, DSA remains the gold standard for diagnosis; although CTA and MRI can be used to identify the PAVF trace, the trace is often the draining vein. In PAVFs, the draining vein can dilate too thicker than the feeding artery, and due to insufficient resolution, the feeding artery may not be identified. When a PAVF creates a high-flow shunt, the affected area of perimedullary veins over the anterior and posterior surfaces of the spinal cord often extends over multiple levels; in the present case, the perimedullary draining vein reached the cervical level (Fig. 2D). Therefore, when searching for the fistula point, it is necessary to examine the whole spinal cord by DSA from the vertebral artery to the iliac artery.

Symptomatic PAVFs should be administered treatment promptly. The currently available treatments for PAVF include surgery, endovascular embolization and a combination of the two methods (12). Regardless of the method used, the fistula must be completely removed or blocked during treatment. For small PAVFs with a simple angioarchitecture, endovascular embolization is considered a beneficial choice. For instance, Cohen et al (1) reported the case of a PAVF in an 8-year-old boy that was supplied by the ADG and a thoracic radiculomedullary artery that joined at the fistula site in a large partially thrombosed varix, which was completely occluded with the liquid embolic material Onyx.

When PAVFs are large and exhibit a high flow, simple surgical removal is associated with certain risks, as it is difficult to determine the fistula angioarchitecture, and it can be difficult to completely occlude the fistula via endovascular embolization (13). Therefore, the optimal choice is the combination of intraoperative DSA and microsurgery. In the case described herein, the selective angiography of the AKA and the ADG was performed, and the feeding arteries were easily identified and resected. Notably, DSA can confirm the completion of treatment.

In the majority of cases of PAVFs, satisfactory outcomes can be achieved after timely treatment, as well as in cases of other arteriovenous shunts, as presented in Table I. In these cases, timely treatment is considered critical in order to avoid an excessive amount of delayed neuronal death. Following surgery, anticoagulation therapy should be administered to avoid postsurgical retrograde thrombosis of the spinal artery and the pial venous plexuses along the spinal cord. Therefore, according to the literature review performed in the present study, microsurgery with intraoperative DSA assistance may be an effective treatment strategy for PAVFs involving the ADG.

\section{Acknowledgements}

Not applicable.

\section{Funding}

No funding was received.

\section{Availability of data and materials}

The datasets used and/or analyzed during the current study are available from the corresponding author upon reasonable request.

\section{Authors' contributions}

YW and JY designed the study and drafted the manuscript. YW collected the data. JY and YW confirm the authenticity of all the raw data. Both authors have read and approved the final manuscript.

\section{Ethics approval and consent to participate}

Ethics approval was not required by the authors' institution, as the present study is a case report. Informed signed consent to participate was obtained from the patient.

\section{Patient consent for publication}

The patient provided consent and agreed for her data to be published.

\section{Competing interests}

The authors declare that they have no competing interests.

\section{References}

1. Cohen JE, Constantini S, Gomori JM, Benifla M and Itshayek E: Pediatric perimedullary arteriovenous fistula of the conus medullaris supplied by the artery of Desproges-Gotteron. J Neurosurg Pediatr 11: 426-430, 2013.

2. Mizutani K, Consoli A, Maria FD, Condette Auliac S, Boulin A Coskun O, Gratieux $\mathbf{J}$ and Rodesch G: Intradural spinal cord arteriovenous shunts in a personal series of 210 patients: Novel classification with emphasis on anatomical disposition and angioarchitectonic distribution, related to spinal cord histogenetic units. J Neurosurg Spine: April 2, 2021 (Epub ahead of print).

3. Reis C, Rocha JA, Chamadoira C, Pereira P and Fonseca J: Foraminal L5-S1 disc herniation and conus medullaris syndrome: A vascular etiology? Acta Neurochir (Wien) 149: 533-535; discussion 535, 2007.

4. Tubbs RS, Mortazavi MM, Denardo AJ and Cohen-Gadol AA: Arteriovenous malformation of the conus supplied by the artery of Desproges-Gotteron. J Neurosurg Spine 14: 529-531, 2011.

5. Seeder L: Muscle strength grading. Ann Emerg Med 12: 407, 1983.

6. Harrow-Mortelliti M, Reddy V and Jimsheleishvili G: Physiology, Spinal Cord. In: StatPearls. StatPearls Publishing, Treasure Island, FL, 2021.

7. Rankin J: Cerebral vascular accidents in patients over the age of 60. II. Prognosis. Scott Med J 2: 200-215, 1957.

8. Rouques L, David M, Desproges and Israel: A new case of traumatic segmental arachnoiditis. Rev Neurol (Paris) 91: 124-126, 1954 (In French).

9. Munich SA, Krishna C, Cress MC, Dhillon GS, Pollina J and Levy EI: Diagnosis and endovascular embolization of a sacral spinal arteriovenous fistula with 'Holo-spinal' venous drainage. World Neurosurg 128: 328-332, 2019. 
10. Ji T, Guo Y, Shi L and Yu J: Study and therapeutic progress on spinal cord perimedullary arteriovenous fistulas. Biomed Rep 7: 214-220, 2017.

11. Gailloud P and Jallo GI: Delayed formation of a symptomatic de novo low-flow perimedullary arteriovenous fistula two years after successful treatment of a high-flow perimedullary arteriovenous fistula. J Neuroradiol 48: 22-24, 2021.

12. Ioannidis I, Nasis N, Plakas S, Chrysicopoulos C and Andreou A: Combined surgical and endovascular approach to treat a ventrally located perimedullary arteriovenous fistula. Childs Nerv Syst 37: 645-648, 2021.
13. Li J, Zeng G, Zhi X, Bian L, Yang F, Du J, Ling F and Zhang H: Pediatric perimedullary arteriovenous fistula: Clinical features and endovascular treatments. J Neurointerv Surg 11: 411-415, 2019.

(i) (3) This work is licensed under a Creative Commons cc) Atribution-NonCommercial-NoDerivatives 4.0 International (CC BY-NC-ND 4.0) License. 